\title{
Crack Growth Signal Processing Approach Combining Wavelet Threshold Denoising and Variable Amplitude DCPD Technique
}

\author{
Chenqiang Ni $\mathbb{D},{ }^{1}$ He Xue $\mathbb{D}^{1},{ }^{1}$ Shuai Wang $\mathbb{D}^{1,2}$ Xiurong Fang $\mathbb{C}^{1},{ }^{1}$ and Hongliang Yang $\mathbb{D}^{3}$ \\ ${ }^{1}$ School of Mechanical Engineering, Xi'an University of Science and Technology, Xi'an 710054, China \\ ${ }^{2}$ Department of Mechanical and Aerospace Engineering, Brunel University London, Uxbridge UB8 3PH, UK \\ ${ }^{3}$ Center of Engineering Training, Xi'an University of Science and Technology, Xi'an 710054, China \\ Correspondence should be addressed to He Xue; xuehe@xust.edu.cn
}

Received 19 May 2021; Revised 21 September 2021; Accepted 5 October 2021; Published 27 October 2021

Academic Editor: Alberto Campagnolo

Copyright $\odot 2021$ Chenqiang Ni et al. This is an open access article distributed under the Creative Commons Attribution License, which permits unrestricted use, distribution, and reproduction in any medium, provided the original work is properly cited.

The direct current potential drop (DCPD) method is widely used in laboratory environments to monitor the crack initiation and propagation of specimens. In this study, an anti-interference signal processing approach, combining wavelet threshold denoising and a variable current amplitude DCPD signal synthesis technique, was proposed. Adaptive wavelet threshold denoising using Stein's unbiased risk estimate was applied to the main potential drop signal and the reference potential signal under two different current amplitudes to reduce the interference caused by noise. Thereafter, noise-reduced signals were synthesized to eliminate the time-varying thermal electromotive force. The multiplicative interference signal was eliminated by normalizing the main potential drop signal and the reference potential drop signal. This signal processing approach was applied to the crack growth monitoring data of $316 \mathrm{~L}$ stainless steel compact tension specimens in a laboratory environment, and the signal processing results of static cracks and propagation cracks under different load conditions were analyzed. The results showed that the proposed approach can significantly improve the signal-to-noise ratio as well as the accuracy and resolution of the crack growth measurement.

\section{Introduction}

The direct current potential drop (DCPD) technique is commonly used to monitor the crack growth rate of metal specimens in laboratory environments [1-4]. It is based on the principle that crack growth in a metal specimen increases its equivalent resistance $[5,6]$. The method involves passing a constant DC to the tested specimens and then observing the potential drop between two points across the crack surface to monitor the crack growth [7]. The relationship between crack growth and DCPD can be obtained from the electric field analysis of a specimen using the finite element method [8-10]. The DCPD signal is significantly small, approximately tens of microvolts to hundreds of microvolts [11]. Because the connections between the measuring leads, specimens, and measuring instruments are often connections of dissimilar materials and because of the existence of temperature difference, the thermal electromotive force (TEMF) is superimposed on the signal. However, owing to the environmental electromagnetic interference and the influence of the instrument itself, the DCPD signal is also accompanied by complex random background noise interference signals. The amplitude of these interference signals accounts for a large part of the effective DCPD signal amplitude; thus, it is difficult to distinguish weak DCPD signals, which significantly affects the measurement accuracy of the signal and the resolution of the crack growth measurement [12-14]. To suppress these interference signals, the reversing DCPD method is used to eliminate the TEMF $[15,16]$. This method requires the use of relay groups in the test circuit, thereby increasing the complexity of the circuit. To suppress the signal drift caused by the temperature drift of the instruments, the reference specimen method or the reference potential method is employed to provide an additional reference DCPD signal to achieve the correction of the main DCPD signal [17]. To the best of our knowledge, no signal processing method has been reported for random noise interference signals on DCPD signals. If 
these random noises are not managed properly, they will inevitably affect the measurement accuracy and resolution. Wavelet analysis is a good time-frequency analysis method, which has the characteristics of multiresolution analysis and has good analysis characteristics in both the time and frequency domains [18]. Therefore, wavelet analysis is suitable for the analysis and processing of weak DCPD signals and other nonstationary signals [19]. The wavelet threshold denoising algorithm is a signal denoising method based on wavelet analysis developed in recent years. It uses wavelet transform to decompose the signal into wavelet coefficients in different frequency bands that can represent the overview and details of the signal, and then processes these coefficients by thresholding methods to achieve denoising [20]. In addition, this algorithm can perform multiresolution analysis of signals and has been applied to many fields, such as geophysics, biomedicine, and vibration analysis in recent years. Compared to other signal processing methods, it has the advantages of multiresolution features and localized features in the time and frequency domains [21]. The wavelet threshold denoising algorithm can also be combined with other signal processing algorithms to achieve a better denoising effect [22, 23].

A signal composition model was proposed in this study to improve the measurement accuracy of the DCPD signal. According to this model, a crack propagation signal processing approach using a wavelet threshold denoising algorithm and variable current amplitude DCPD signal synthesis technique is proposed. The $316 \mathrm{~L}$ stainless steel compact tension $(\mathrm{C}(\mathrm{T})$ ) specimens were selected to conduct DCPD signal monitoring experiments under static and dynamic crack growth conditions. The validity and practicability of the signal model and signal processing approaches were verified based on experimental data. The proposed approach helps to further improve the measurement accuracy of the crack growth monitoring DCPD signal and the measurement resolution of crack growth.

\section{Experiment}

Two $\mathrm{C}(\mathrm{T})$ specimens were used for the fatigue tensile and DCPD monitoring experiments in an ambient laboratory air environment. The ambient temperature range was $24-30^{\circ} \mathrm{C}$. The geometry and dimensions of the $\mathrm{C}(\mathrm{T})$ specimens are shown in Figure 1. The material used as the specimens was $316 \mathrm{~L}$ stainless steel. The initial crack lengths $a_{0}$ of specimens $\# 1$ and \#2 were $10 \mathrm{~mm}$ and $12 \mathrm{~mm}$, respectively. For specimen $\# 2$, it was cut with a width of $0.2 \mathrm{~mm}$ and a length of $2 \mathrm{~mm}$ slit at the notch root along the ligament plane by wire cutting to simulate cracks.

In the DCPD crack growth monitoring test, the specimen was injected with a constant current, and the DCPD signals between specific positions were monitored. The measured DCPD signals include the main DCPD signal $V$ and the reference DCPD signal $V_{r}$. The current lead positions $\left(I+, I_{-}\right)$and the DCPD signal $\left(V+, V-, V_{r}+, V_{r^{-}}\right)$lead positions of the specimens are shown in Figure 2.

The crack growth monitoring experimental system based on the DCPD technique is shown in Figure 3. The experimental equipment included a tensile testing machine and a

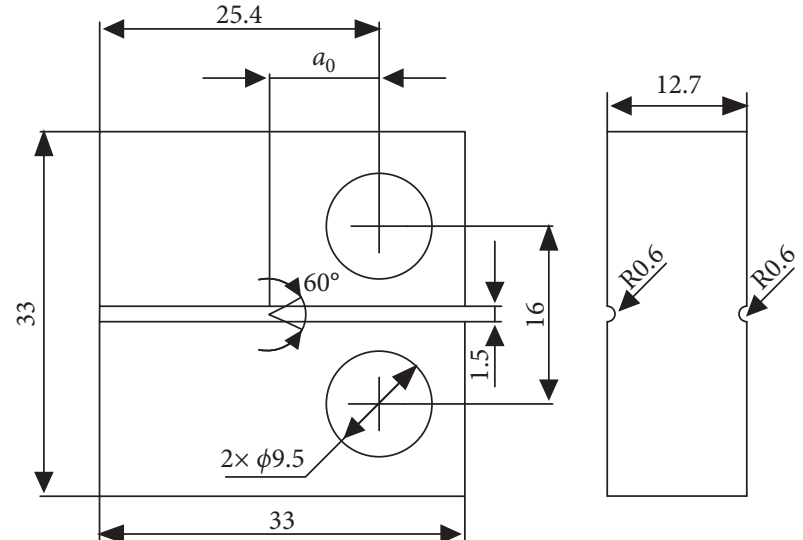

Figure 1: Geometry and dimensions of the $\mathrm{C}(\mathrm{T})$ specimens.

set of DCPD crack growth monitoring system. The tensile testing machine can apply static or dynamic tensile loads to the specimen and can conduct fatigue precracking and crack growth experiments on specimens. Fatigue tensile tests with different frequencies and loads were performed using a tensile testing machine. During the test, the DCPD of the specimen was monitored using a DCPD crack growth monitoring system.

The composition and connections of the DCPD crack growth monitoring system are shown in Figure 4 . It consists of a computer, high-performance power supply, nanovoltmeter, data-acquisition switch instrument, and an ambient temperature measurement module. The ambient temperature measurement module was connected to a computer through an RS232 serial interface. Other instruments were interconnected through a general-purpose interface bus (GPIB) that was connected to a computer through a physical cell identifier-general-purpose interface bus (PCI-GPIB) interface card. These instruments work together under the control of software running on a computer. During the tensile test of the specimens, a power supply was used to provide a constant current for the specimens. It has low current ripple and noise as well as a high output current programming resolution. The output current range is $0-5 \mathrm{~A}$, which can be dynamically set by the control software. A nanovoltmeter was used to measure the signal of a small potential drop. The main and reference DCPD signals of the specimen were connected to the input channel of the data-acquisition switch instrument, and the output channel was connected to the measurement channel of the nanovoltmeter. During the test, the main control software controls the data-acquisition switch to switch the measured signals. Another software running on the computer records the ambient temperature during the test through the ambient temperature measurement module. The recorded ambient temperature data were used to analyze the temperaturerelated interference signals.

The wiring and installation of the $\mathrm{C}(\mathrm{T})$ specimen on the testing machine are shown in Figure 5. To insulate the specimen from the tensile testing machine, zirconia ceramic tubes were used to separate the specimen loading hole and 


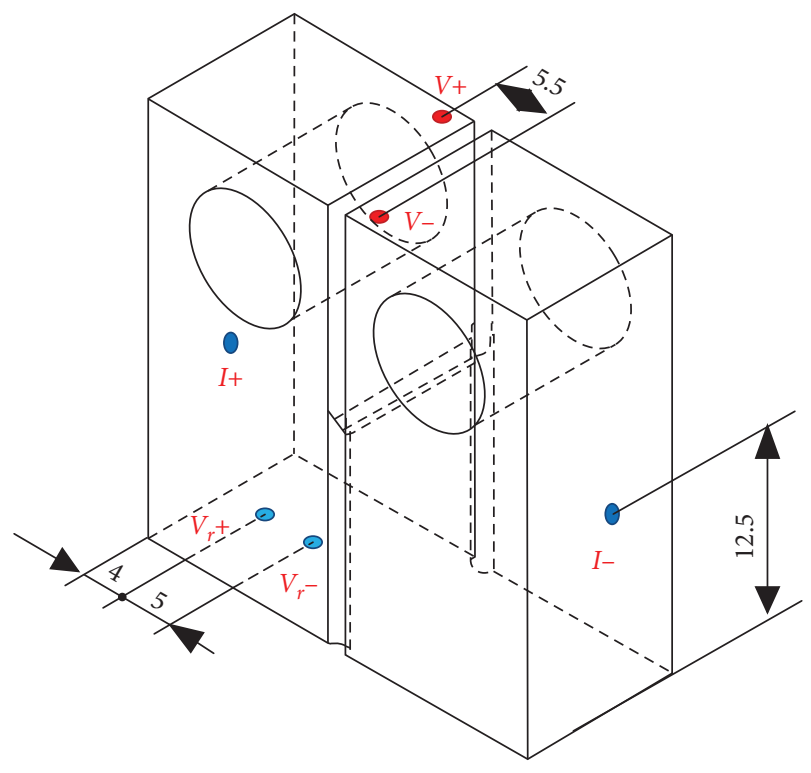

FIGURE 2: Lead positions of the $\mathrm{C}(\mathrm{T})$ specimens.

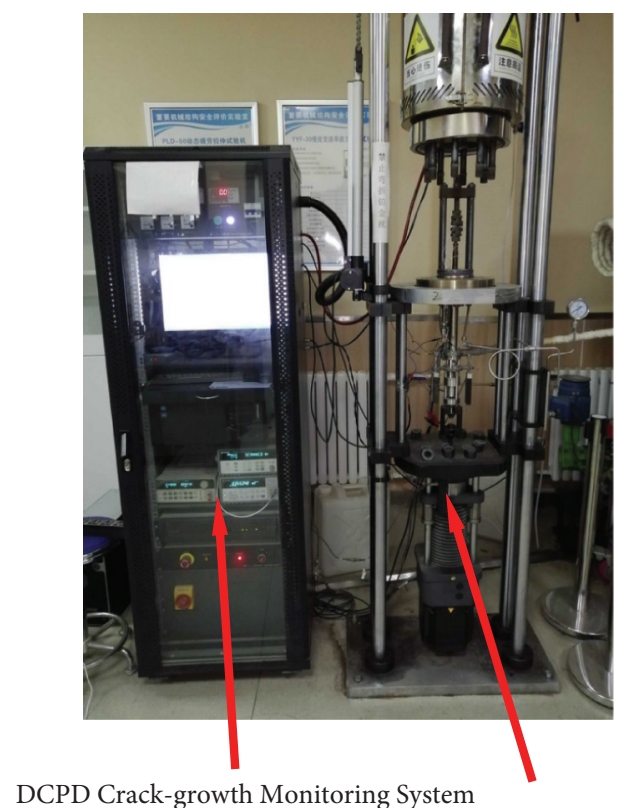

Tensile Testing Machine

Figure 3: Test setup.

the pin, and zirconia ceramic sheets were used to separate the specimen side from the fixture. Both the current and DCPD signal leads used were platinum wires with diameters of $0.75 \mathrm{~mm}$ and $0.5 \mathrm{~mm}$, respectively. They were connected to the specimen via spot welding. The leads were covered with a polytetrafluoroethylene (PTFE) tube to ensure insulation. In addition, they were connected to the test instrument through twisted wire pairs after being drawn from the specimen.

\section{DCPD Signal Model and Anti-Interference Signal Processing Approach}

3.1. DCPD Signal Model. Owing to the good conductivity of the metal specimens and the limitation of the input current amplitude, the DCPD signal is only at the microvolt level. Several factors influence the DCPD signal, including environmental electromagnetic interference, instrumentation drift, TEMF, noise from the mains, crack surface contact, and specimen deformation under load $[1,7,13]$. The influence of these factors on the DCPD signal is that different types of interference signals are superimposed on the measured signal. Figure 6 shows an example of the measured DCPD signals (specimen $\# 1$, current $I=0.28 \mathrm{~A}$, sampling frequency $f_{s}=0.385 \mathrm{~Hz}$ ) when the specimens were not loaded (i.e., static crack). These interference signals should be removed before they are converted into crack growth signals. According to our experimental data of the $\mathrm{C}(\mathrm{T})$ specimens and the reports in the literature $[1,7,13]$, the DCPD signal contains the following types of interference signals:

(1) TEMF interference signal: TEMF is generated when two junctions of dissimilar metals are maintained at different temperatures. In particular, the wire materials used in the DCPD measurement are not of the same material as the specimen or connectors on the measuring instruments. Any difference in temperature between the junctions generates the TEMF [24]. This type of interference signal has an additive relationship with real signals. Because it is related to temperature, this interference signal is not static and will change with the change in the complex temperature field distribution in the environment. During the test, the temperature fluctuation of the specimen and the fluctuation of the ambient temperature caused the fluctuation of the TEMF.

(2) Multiplicative interference signals: This type of signal originates from the temperature drift of the semiconductor device of the measuring instruments, which is difficult to eliminate. This is caused by the change in the characteristics of the measurement channel with temperature. It is characterized by a temperature coefficient and has a multiplicative relationship with the measured signal. According to the experimental data of different current amplitudes under static crack conditions, it can be assumed that the amplitude is approximately linear with the real signal amplitude.

(3) Random noise signal: The sources of random noise signals are complex, including interference from the mains and loading system, as well as the conversion noise of the A/D device of the instrument. For this type of noise signal with complex sources, it appears as flat broadband characteristics; thus, it can be approximately regarded as additive white Gaussian noise [25-27]. 


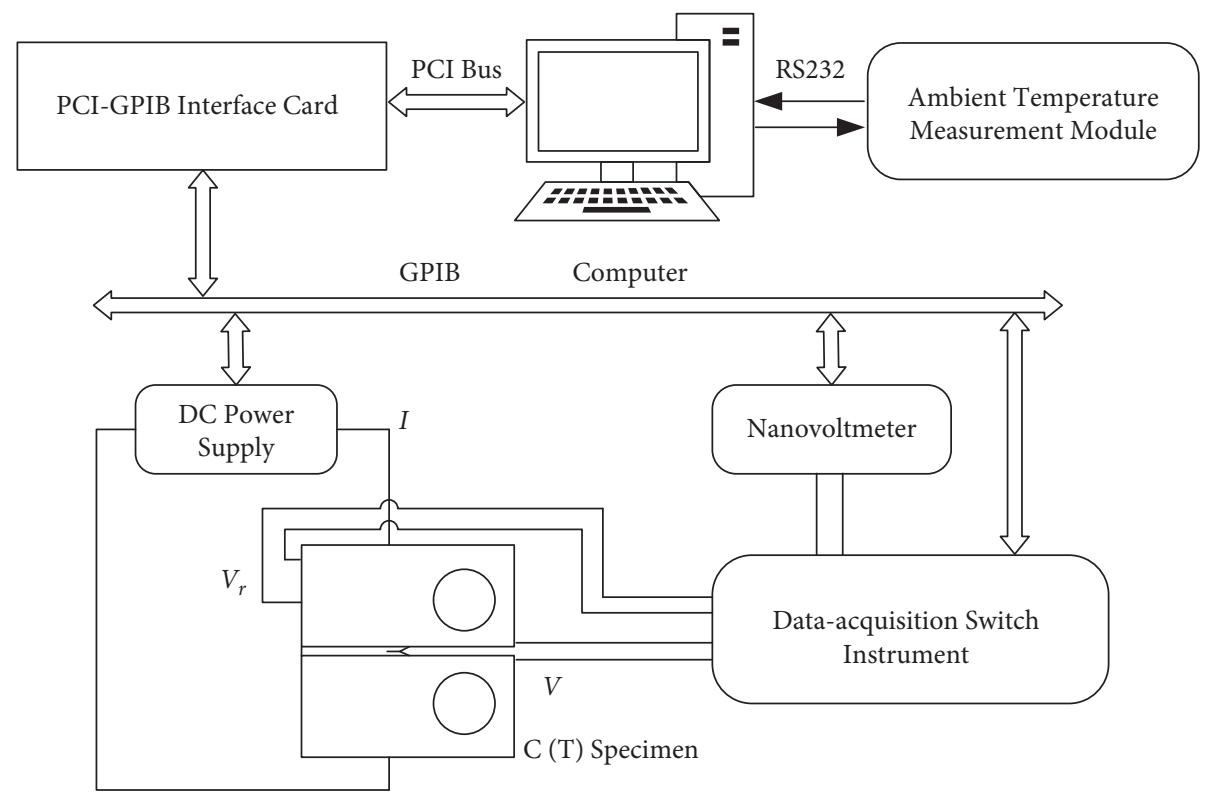

FIGURE 4: Composition and connections of the DCPD crack growth monitoring system.

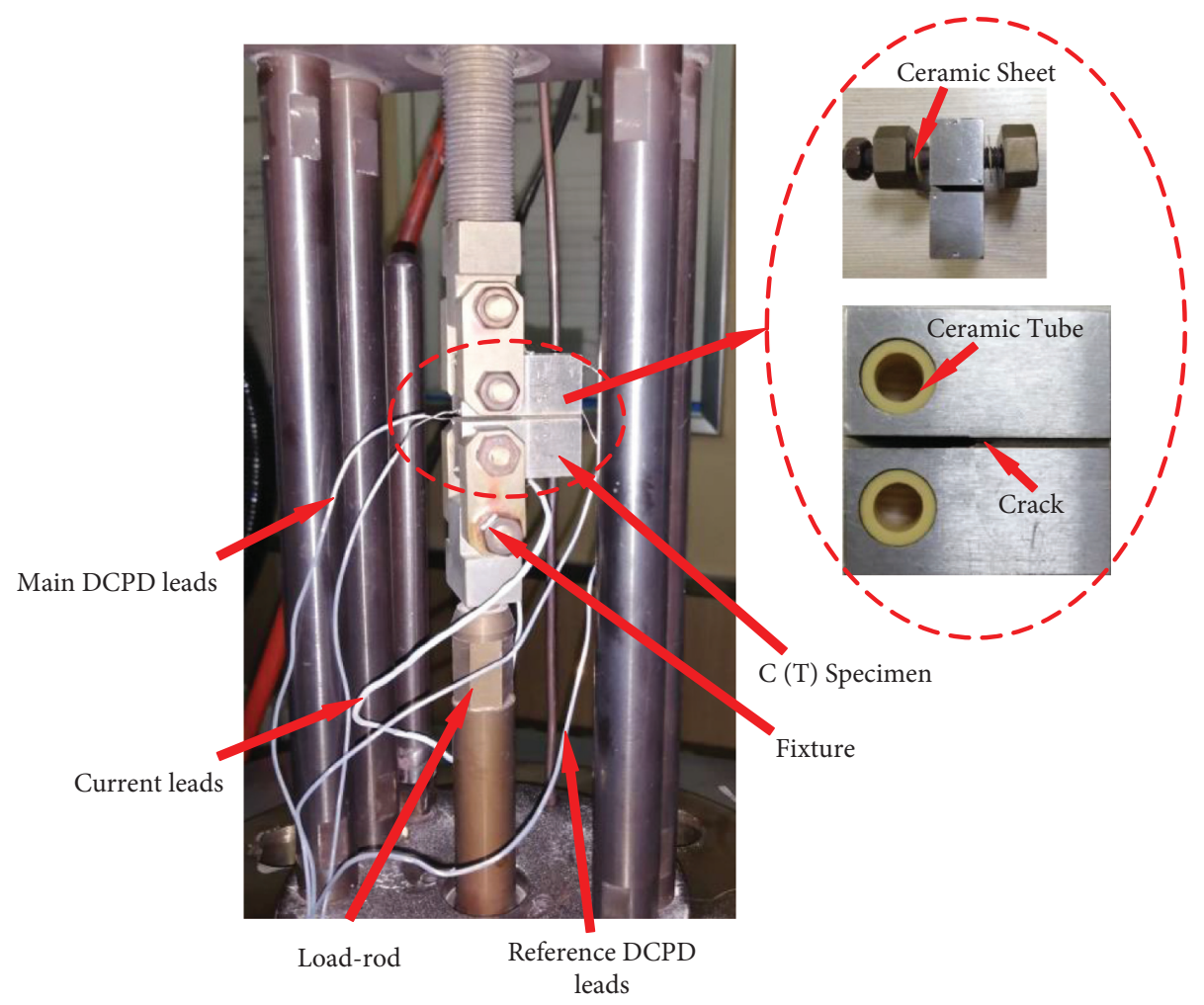

Figure 5: Installation and wiring of the $\mathrm{C}(\mathrm{T})$ specimen.

According to the above characteristics of the DCPD signal, the DCPD signal model can be expressed as follows:

$$
V(t, a)=V^{*}(a)+C(t)+D(t, a)+n(t)
$$

where $t$ is time, $a$ is the crack length, $V$ is the measured DCPD signal, $V^{*}$ is the true value of the DCPD signal, which is a function of the crack length $a, C$ is the TEMF interference signal, $D$ is the multiplicative interference signal, and $n$ is the random noise signal.

3.2. Adaptive Wavelet Threshold Denoising of DCPD Signals. The purpose of the adaptive wavelet threshold denoising of the DCPD signal is to reduce the random noise signal $n(t)$, which has a wider frequency band compared with other 


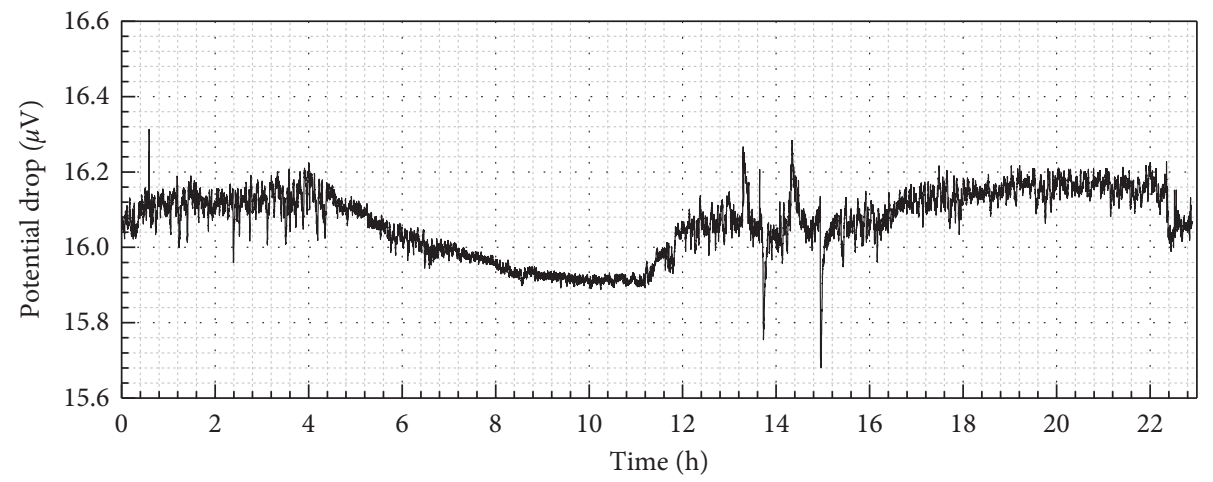

FIgURE 6: Measured DCPD signal at the static crack (specimen $\# 1$, current $I=0.28$ A sampling frequency $f_{s}=0.385 \mathrm{~Hz}$ ).

types of interference signals. For the noisy signals, after wavelet transformation was performed on the signal, in the wavelet domain, the coefficient corresponding to the effective signal became large, whereas the coefficient corresponding to the noise became significantly small. The statistical properties of the coefficients corresponding to the noise in the wavelet domain were consistent with the original noise. The coefficient amplitude of the useful signal was greater than the coefficient amplitude of the noise. Therefore, the wavelet coefficients at each scale can be obtained through wavelet decomposition. Thereafter, the signal and noise can be distinguished by setting a reasonable threshold to achieve signal denoising [28]. Although wavelet threshold denoising can be highly regarded as a low-pass filter, it is superior to the traditional low-pass filter. Wavelet threshold denoising can better retain the characteristics of the real signal itself while denoising, such as some local relatively fast change characteristics of the real signal itself. Therefore, the use of wavelet threshold denoising allows better retention of the characteristics of the real signal itself and other types of interference signals while denoising. Thus, the targeted processing of other types of interference signals cannot be affected. The wavelet threshold denoising process is conducted according to the following three steps [29]:

(1) Perform a wavelet transform on a noisy signal. Choose a wavelet basis and determine the level $N$ of the wavelet decomposition, and then perform $\mathrm{N}$ level wavelet decomposition on the signal.

(2) Obtain threshold wavelet coefficients. To maintain the overall shape of the signal unchanged and retain the effective signal, the coefficients of each layer after decomposition are quantized by a hard threshold, soft threshold, or other threshold methods.

(3) Perform an inverse wavelet transform to reconstruct the signal.

In this study, the $\mathrm{db} 4$ wavelet was selected as the wavelet base, and the signal to be processed was decomposed into four layers to obtain the wavelet coefficients. Thereafter, the threshold of wavelet coefficients was obtained, and the signal was reconstructed. The selection of the threshold directly affects the denoising effect. Stein's unbiased risk estimate (SURE) was selected. It is an adaptive threshold selection method with minimum risk as an indicator. The specific algorithm is as follows:

(1) After wavelet decomposition, the absolute value of the wavelet coefficients of each layer $\omega_{k, j}$ is sorted from small to large, and then the absolute value of the wavelet coefficients after sorting is squared to obtain the $k$-th layer vector:

$$
\begin{aligned}
S_{k} & =\left[s_{k, 1}, s_{k, 2}, \ldots, s_{k, N}\right], \\
s_{k, j} & =\left(\operatorname{sort}\left(\left|\omega_{k, j}\right|\right)\right)^{2},
\end{aligned}
$$

where $N$ is the number of wavelet coefficients in each layer.

(2) Calculate the threshold vector $\lambda_{k}$ of the $k$-th layer as

$$
\begin{aligned}
\lambda_{k} & =\left[\lambda_{k, 1}, \lambda_{k, 2}, \ldots, \lambda_{k, N}\right], \\
\lambda_{k, j} & =\sqrt{s_{k, j}} .
\end{aligned}
$$

(3) The risk estimation coefficient vector $R_{k}$ generated when $\lambda_{k, j}$ is selected on the $k$ th layer is

$$
\begin{aligned}
r_{k, j} & =\frac{\left[N-2 j+\sum_{i=1}^{j} s_{k, i}+(N-j) s_{k, N-j}\right]}{N}, \\
R_{k} & =\left[r_{k, 1}, r_{k, 2}, \ldots, r_{k, N}\right] .
\end{aligned}
$$

(4) By comparing the vector of the risk estimation coefficients, the unbiased risk estimation threshold of the $k t h$ layer decomposition is

$$
\lambda_{k \_b e s t}=\sqrt{s_{k, j_{\min }}},
$$

where $j_{\min }$ is the $j$ corresponding to the minimum risk assessment coefficient.

The threshold function is a rule for modifying wavelet coefficients, and different threshold functions reflect different strategies for processing the wavelet coefficients. The threshold processing function of wavelet threshold denoising is divided into a soft threshold function and a hard threshold function. In this study, the soft threshold function was used to reduce the noise of the DCPD signal. The soft threshold function is 


$$
\bar{\omega}_{k, j}= \begin{cases}0, & \left|\omega_{k, j}<\lambda_{k}\right|, \\ \operatorname{sign}\left(\omega_{k, j}\right)\left(\left|\omega_{k, j}\right|-\lambda\right), & \left|\omega_{k, j} \geq \lambda_{k}\right|,\end{cases}
$$

where $\bar{\omega}_{k, j}$ are the wavelet coefficients obtained after threshold processing, sign is the sign function, and $\lambda_{k}$ is the threshold of the kth layer.

\subsection{Variable Current Amplitude DCPD Signal Synthesis} Technique. The reversing DCPD method was used for the TEMF interference signal. However, owing to the relay group introduced in the circuit, the complexity of the circuit increased, and additional interference signals were introduced. After the relay was switched, it was necessary to wait for the signal to stabilize before measuring; thus, the measurement speed was also affected. A new method of changing the current amplitude was adopted to eliminate the TEMF interference signal. In this method, two sets of DCPD signals were obtained by controlling the program-controlled constant current source to continuously output two different amplitude currents and then combining them to eliminate the TEMF. Consider the case in which the signal contains only multiplicative interference and TEMF. When the input currents are $I 1$ and $I 2$, the measured signal can be expressed as

$$
\begin{aligned}
& V_{I 1}(t, a)=V_{I 1}^{*}(a)+C_{I 1}(t)+D_{I 1}(t, a), \\
& V_{I 2}(t, a)=V_{I 2}^{*}(a)+C_{I 2}(t)+D_{I 2}(t, a),
\end{aligned}
$$

where $V_{I 1}$ and $V_{I 2}$ are the measured values of the signal when the input currents are $I 1$ and $I 2$, respectively; $V_{I 1}^{*}$ and $V_{I 2}^{*}$ are the true values of the signal; $C_{I 1}$ and $C_{I 2}$ are the TEMF; $D_{I 1}$ and $D_{I 2}$ are the multiplicative interference signals. Owing to the short interval between the two currents,

$$
C_{I 1}(t) \approx C_{I 2}(t) .
$$

Therefore, the TEMF interference signal is eliminated after the difference between $V_{I 1}$ and $V_{I 2}$ as follows:

$$
V_{I 1}(t, a)-V_{I 2}(t, a)=V_{I 1}^{*}(a)+D_{I 1}(t, a)-V_{I 2}^{*}(a)-D_{I 2}(t, a) \text {. }
$$

Because the DCPD signal is proportional to the magnitude of the current, the multiplicative interference signal is proportional to the magnitude of the DCPD signal:

$$
\frac{D_{I 1}(t, a)}{D_{I 2}(t, a)}=\frac{V_{I 1}^{*}(a)}{V_{I 2}^{*}(a)}=\frac{I_{1}}{I_{2}} .
$$

Express the ratio of the two currents as

$$
k_{c}=\frac{I_{2}}{I_{1}} .
$$

The signal after removing TEMF is

$$
V_{S Y N}=V_{I 1}^{*}(a)+D_{I 1}(t, a)=\frac{V_{I 1}(t, a)-V_{I 2}(t, a)}{\left(1-k_{c}\right)} \text {. }
$$

Therefore, according to the signal measured under two consecutive different current signals and the ratio $k_{c}$ of the two currents, the DCPD signal without the TEMF signal can be obtained, and the multiplicative interference signal is retained.

3.4. Elimination Method of Multiplicative Interference Signal. As shown in Figure 2, to eliminate the multiplicative interference signal, the main DCPD signal $V$ was measured, and the DCPD between two points on the same side of the specimen crack surface was also measured as a reference DCPD signal $V_{r}$. The dimensionless parameter $V_{\text {norm }} \mathrm{ob}-$ tained by the normalization of the two signals was used as the final parameter for calculating the crack length. The expression for $V_{\text {norm }}$ is

$$
\begin{aligned}
V_{\text {norm }}(a) & =\frac{V^{*}(a) / V_{0}}{V_{r}^{*}(a) / V_{0}}=A \frac{V^{*}(a)}{V_{r}^{*}(a)}, \\
A & =\frac{V_{r 0}}{V_{0}},
\end{aligned}
$$

where $V_{o}$ and $V_{r 0}$ are the initial main DCPD and the initial reference DCPD, respectively, which are determined by the initial crack length and the lead positions, and are measured before crack propagation; $V^{*}$ and $V_{r}^{*}$ are the true values; and $A$ is a constant determined by $V_{o}$ and $V_{r 0}$. The calibration function can be obtained using a three-dimensional electrical finite element analysis.

Considering that there is only a multiplicative interference signal in the signal, the main DCPD signal and the reference DCPD signal can be expressed as

$$
\begin{aligned}
V(t, a) & =V^{*}(a)+D(t, a), \\
V_{r}(t, a) & =V_{r}^{*}(a)+D_{r}(t, a),
\end{aligned}
$$

where $D$ and $D_{r}$ are the multiplicative interference signals of the two signals, respectively. Because the multiplicative interference signal is proportional to the real signal, the multiplicative interference signal can be expressed as

$$
\begin{aligned}
D(t, a) & =\alpha(t) V^{*}(a), \\
D_{r}(t, a) & =\alpha(t) V_{r}^{*}(a),
\end{aligned}
$$

where $\alpha$ is a coefficient related to the parameters of the measuring instrument and the ambient temperature. Thus,

$$
\begin{gathered}
\frac{V^{*}(a)}{V_{r}^{*}(a)}=\frac{V(t, a)}{V_{r}(t, a)}, \\
V_{\text {norm }}(a)=A \frac{V(t, a)}{V_{r}(t, a)} .
\end{gathered}
$$

It can be observed that using $V_{\text {norm }}$ as the parameter for calculating the crack length can eliminate the multiplicative interference signal.

\section{Results and Discussion}

4.1. Signals of Static Crack. The proposed signal processing approach was used to process the main DCPD signal and the reference DCPD signal when the specimen was unloaded (i.e., 


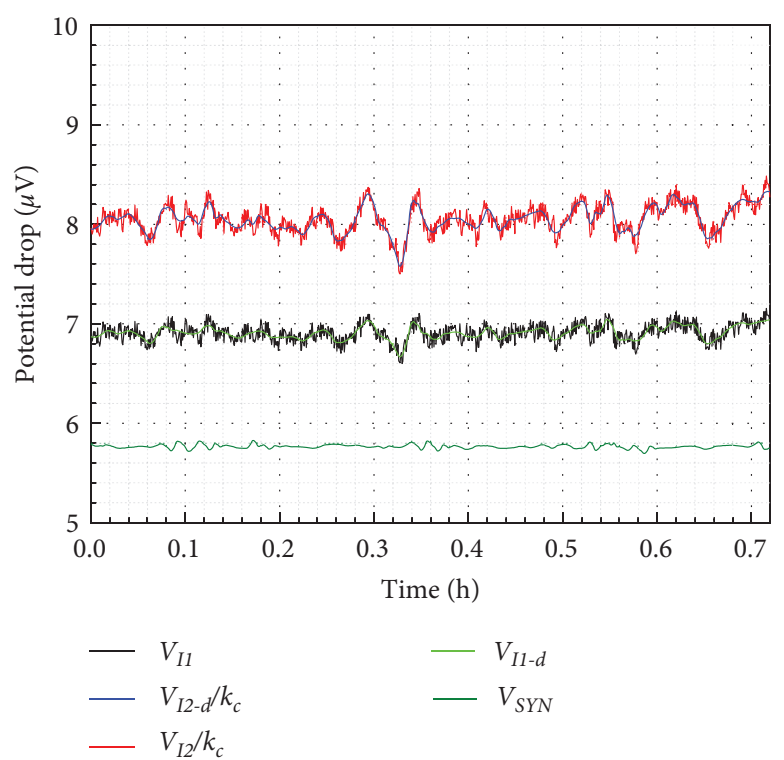

(a)

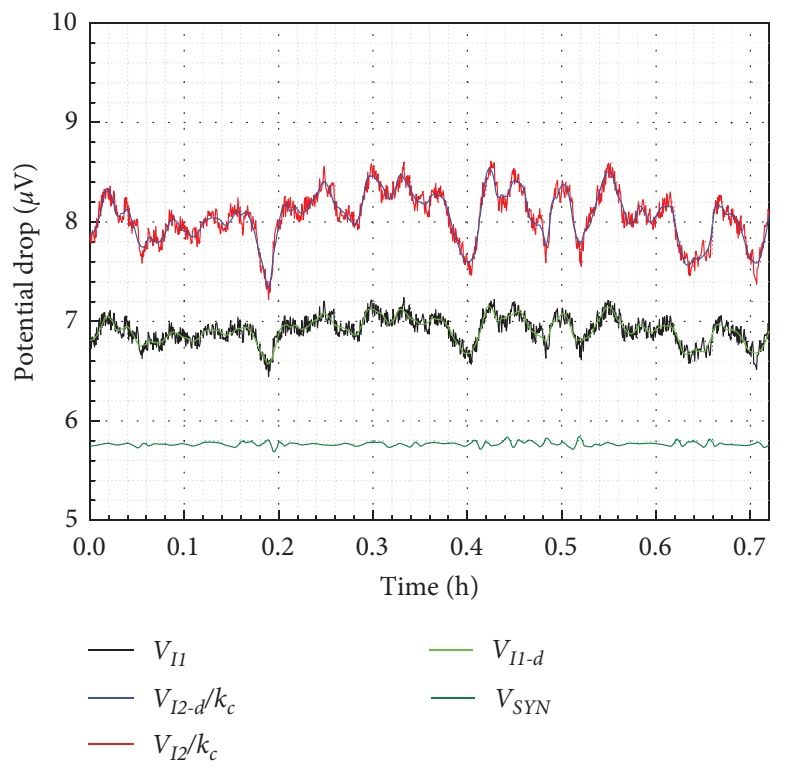

(c)

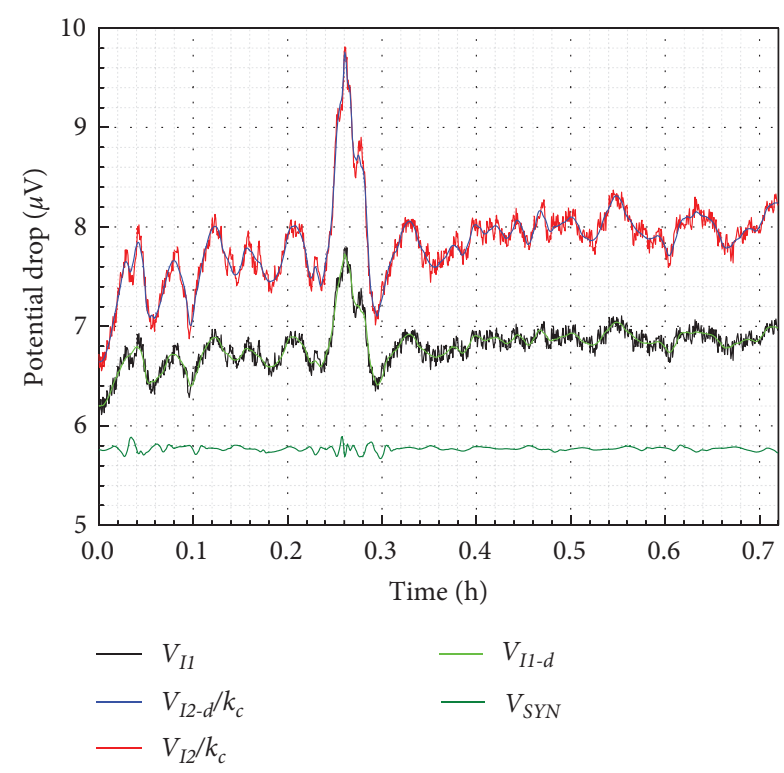

(b)

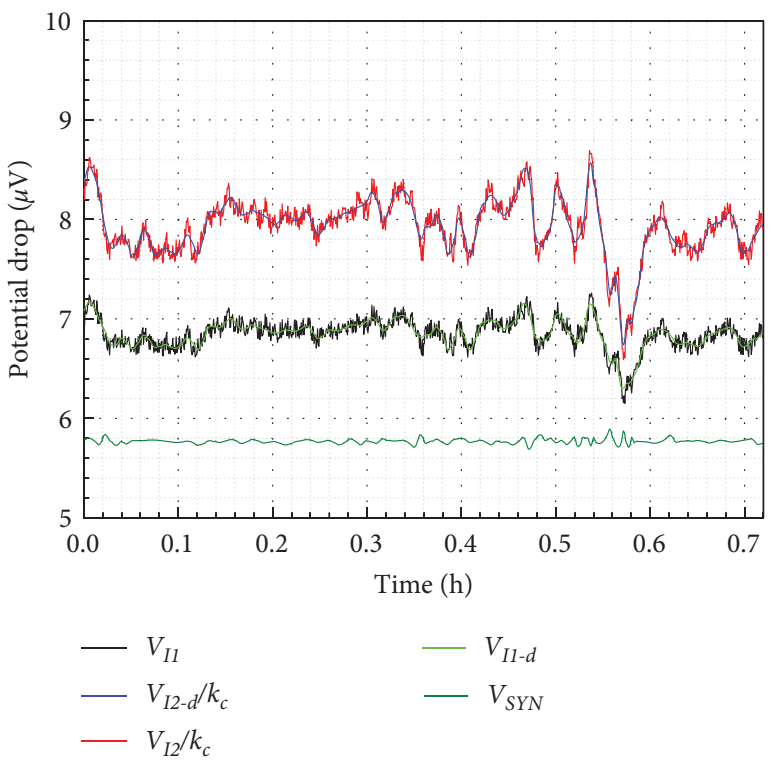

(d)

FIGURE 7: Signal processing results of the main DCPD signal under static crack conditions (specimen \#1, current $I=0.1$ A sampling frequency $f_{s}=0.385 \mathrm{~Hz}$ ).

static crack), and four sets of signals were randomly selected to illustrate the effect of the signal processing approach (specimen \#1, current $I=0.1 \mathrm{~A}$, sampling frequency $f_{s}=0.385 \mathrm{~Hz}$ ). The signals shown in Figure 7 are the main DCPD signals, and the signals shown in Figure 8 are the reference DCPD signals. In Figures 7 and $8, V_{I I-d}$ and $V_{I 2-d}$ are the signals of $V_{I 1}$ and $V_{I 2}$, respectively, after wavelet threshold denoising. To facilitate comparison, $V_{I 2}$ and $V_{I 2-d}$ were divided by $k_{c}$. It can be observed that after wavelet threshold denoising, the noise on signals $V_{I 1}$ and $V_{I 2}$ is significantly reduced while retaining the change characteristics of the lower frequency signals. The signal $V_{S Y N}$ is the signal obtained by the signal synthesis algorithm in equation (12). Moreover, it can be observed that the time-varying TEMF signal is eliminated, and the signal becomes more stable.

To further observe the effect of the signal processing approach, the signal-to-noise ratio (SNR) and root mean square error (RMSE) were used to quantitatively evaluate the performance of the algorithm. The calculation formulas are as follows: 


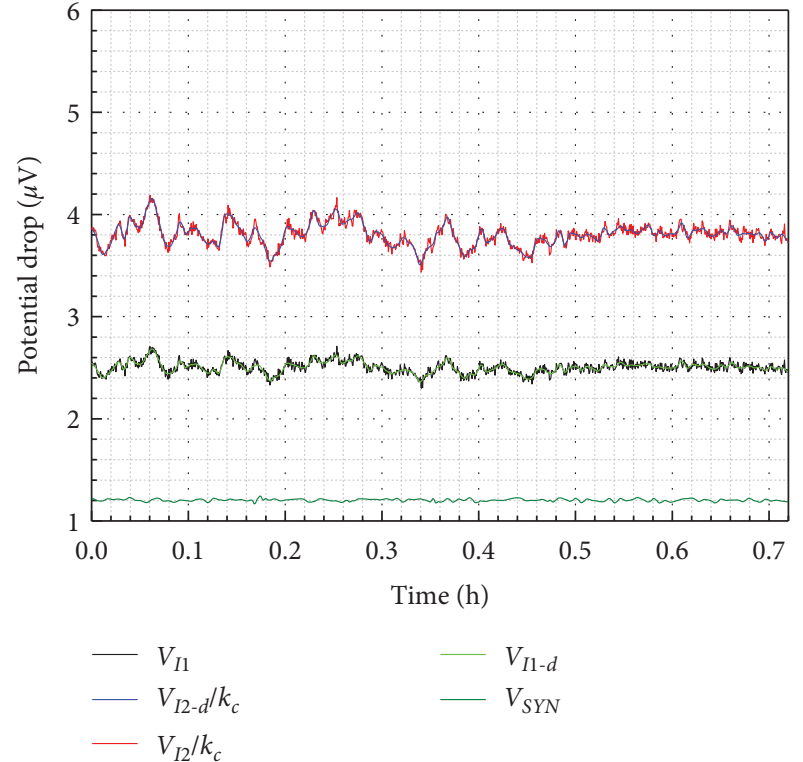

(a)

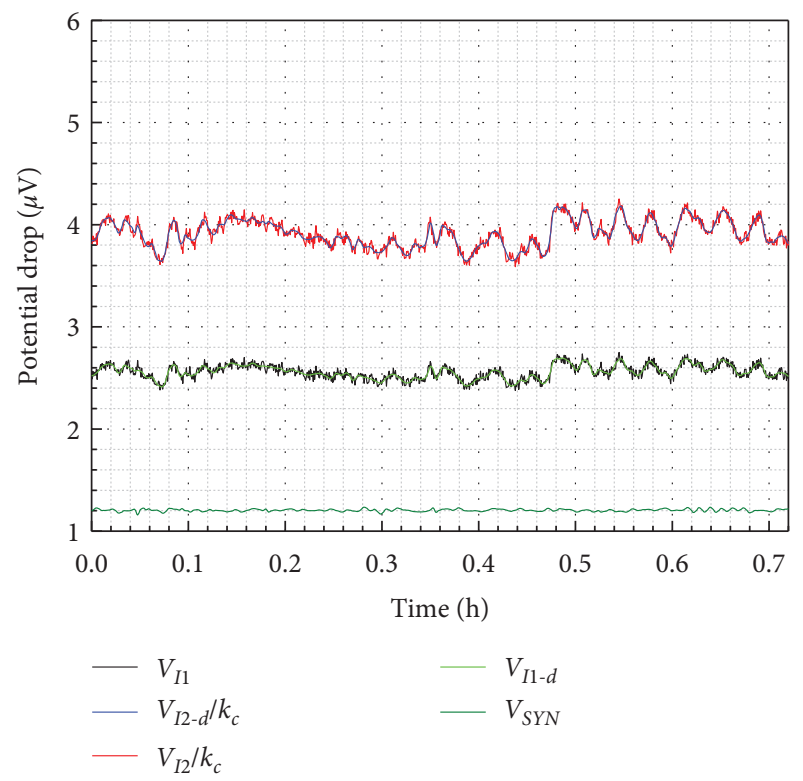

(c)

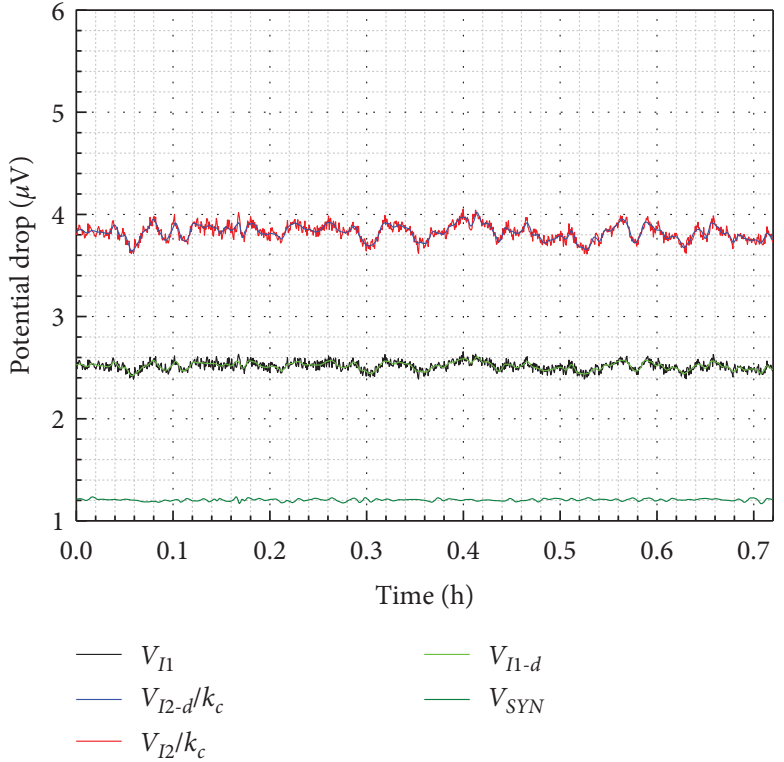

(b)

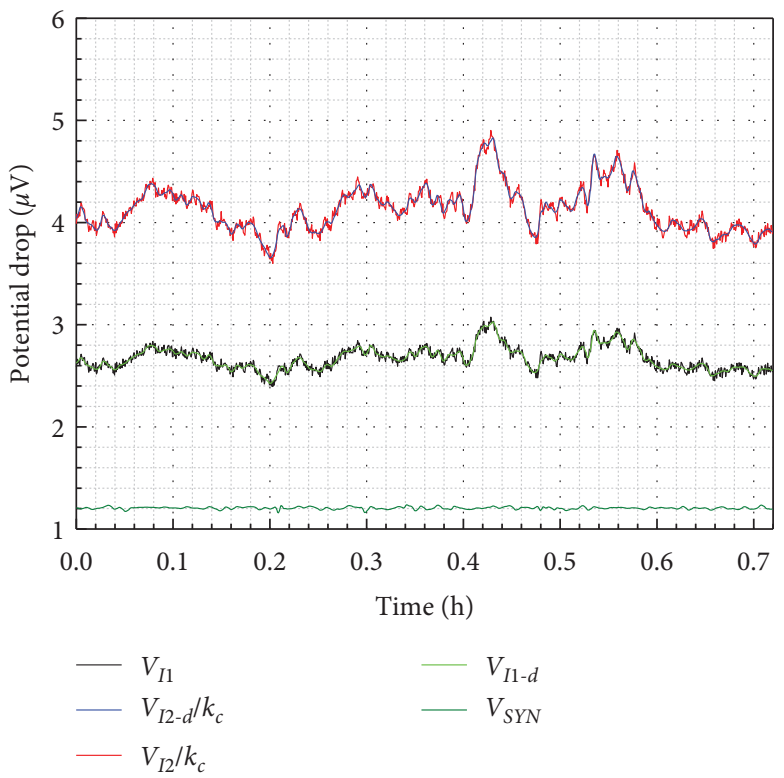

(d)

FiguRE 8: Signal processing results of the reference DCPD signals under static crack conditions (specimen \# 1 , current $I=0.1$ A sampling frequency $f_{s}=0.385 \mathrm{~Hz}$ ).

$$
\begin{aligned}
\mathrm{SNR} & =10 \log \frac{N\left[\operatorname{mean}\left(s_{d}\right)\right]^{2}}{\sum_{i=1}^{N}\left[s(i)-\operatorname{mean}\left(s_{d}\right)\right]^{2}}, \\
\mathrm{RMSE} & =\sqrt{\frac{1}{N} \sum_{i=1}^{N}\left(s(i)-\operatorname{mean}\left(s_{d}\right)\right)^{2}},
\end{aligned}
$$

where $N$ is the number of signal sampling points, $s$ is the signal to be evaluated, $s_{d}$ is the signal processed by the proposed signal processing approach, and mean is the mean value function. Considering that the signal is obtained under static crack conditions, the mean value of $s_{d}$ is used as an estimate of the true signal. The larger the $S N R$ and the smaller the RMSE, the more effective is the signal processing algorithm. Tables 1 and 2 list the RMSE and SNR of the main DCPD signal and the reference DCPD signal before and after the signal processing approach, respectively. It can be observed that the RMSE is reduced by approximately 10 times, and the $S N R$ is increased by approximately $30 \mathrm{~dB}$.

To estimate the crack length RMSE corresponding to the RMSE of the DCPD signal (RMSE_a), using the commercial FE software ABAQUS v6.12, the finite element model of the specimens used in the experiment was established. Finite 
TABLE 1: RMSE, SNR, and RMSE_a of the main DCPD signal before and after signal processing.

\begin{tabular}{lcccc}
\hline Data number & Signal & RMSE $(\mu \mathrm{V})$ & SNR $(\mathrm{db})$ & RMSE_a $(\mathrm{mm})$ \\
\hline \multirow{2}{*}{1} & $V_{I 1}$ & 0.22 & 16.12 & 0.53 \\
& $V_{S Y N}$ & 0.03 & 16.34 & 0.07 \\
\hline \multirow{2}{*}{2} & $V_{I 1}$ & 0.12 & 49.43 & 0.29 \\
& $V_{S Y N}$ & 0.02 & 15.52 & 0.05 \\
\hline \multirow{2}{*}{3} & $V_{I 1}$ & 0.13 & 48.53 & 0.32 \\
& $V_{S Y N}$ & 0.02 & 15.94 & 0.05 \\
\hline \multirow{2}{*}{4} & $V_{I 1}$ & 0.16 & 47.07 & 0.39 \\
& $V_{S Y N}$ & 0.02 & & 0.05 \\
\hline
\end{tabular}

TABLE 2: RMSE and SNR of the reference DCPD signal before and after signal processing.

\begin{tabular}{lccc}
\hline Data number & Signal & RMSE $(\mu \mathrm{V})$ & SNC $(\mathrm{db})$ \\
\hline \multirow{2}{*}{1} & $V_{I 1}$ & 0.06 & 5.68 \\
& $V_{S Y N}$ & 0.01 & 40.23 \\
\hline \multirow{2}{*}{2} & $V_{I 1}$ & 0.05 & 5.66 \\
& $V_{S Y N}$ & 0.01 & 40.18 \\
\multirow{2}{*}{3} & $V_{I 1}$ & 0.07 & 5.52 \\
& $V_{S Y N}$ & 0.01 & 40.46 \\
\multirow{2}{*}{4} & $V_{I 1}$ & 0.11 & 5.19 \\
& $V_{S Y N}$ & 0.01 & 40.51 \\
\hline
\end{tabular}

element electrical analysis of the specimens with six crack lengths was performed. The initial crack length was $10 \mathrm{~mm}$, which increased in increments of $1.016 \mathrm{~mm}$. According to the DCPD analysis results obtained under different crack lengths, the calibration function obtained by polynomial fitting is

$$
\Delta a=0.061(\Delta V)^{3}-0.449(\Delta V)^{2}+2.493(\Delta V),
$$

where $\Delta a$ is the increment of the crack length relative to the initial crack length and $\Delta V$ is the increment of the DCPD. The RMSE values in Table 1 were substituted into equation (18), and the calculated $\Delta a$ was used as the estimated value of RMSE_ $a$, which is also listed in Table 1 . It can be observed that the signal processing approach can increase the resolution of the crack growth measurement approximately by an order of magnitude.

4.2. Signals after the Specimen Is Loaded. Owing to the effect of the resistance strain, the DCPD signal changes with the size of the crack and slightly changes with the deformation of the specimen under load. To evaluate the effect of the signal processing approach on changing signals, the testing machine was set to load control mode and a slow sine wave load was applied to the specimen to generate a slowly and periodically changing DCPD signal. Three sine wave loads with peaks of $2000 \mathrm{~N}, 5000 \mathrm{~N}$, and $8000 \mathrm{~N}$ were used, and the loading frequency was $0.01 \mathrm{~Hz}$. After each load slowly increased to its peak value, it changed according to the sinusoidal rule based on the set frequency. The loading time for each load was approximately five loading cycles. After each loading program segment, the next loading program segment did not start immediately. The force was maintained at the minimum value for the same time to generate a no-load time period, which separated the loading program segments.

When the specimen was subjected to a sinusoidal load, the elastoplastic strain in the crack tip area of the specimen caused a small change, which was approximately linear with the load, in the DCPD signal. This caused the DCPD signal to produce regular sine wave fluctuations. Figure 9 shows the DCPD signals during loading (specimen \#2, current $I=4.0 \mathrm{~A}$, sampling frequency $f_{s}=1.295 \mathrm{~Hz}$ ). It can be observed that before the signal is processed, the sine wave law cannot be distinguished from the measured signal owing to interference signals, such as noise and TEMF. After signal processing, the signal shows an obvious sinusoidal law, which shows that the signal processing approach still has a good anti-interference effect on dynamic weakly changing signals.

4.3. Signals in the Process of Crack Growth. To observe the effect of the proposed signal processing approach during the crack growth process, the specimen was loaded according to the sine wave law at a frequency of $0.1 \mathrm{~Hz}$ to ensure slow growth of the crack. Figure 10 shows a section of the crack propagation signals when the peak force is $8000 \mathrm{~N}$ and the valley force is $6400 \mathrm{~N}$ (specimen \#2, current $I=4.0 \mathrm{~A}$, sampling frequency $f_{s}=3.547 \mathrm{~Hz}$ ). Comparing the DCPD signal measured during the crack growth process with the processed signal, it can be observed that the processed signal reflects both the process of slow crack growth and the small signal fluctuations caused by load changes.

Longer crack propagation signals at a loading frequency of $0.1 \mathrm{~Hz}$ with a peak force of $8000 \mathrm{~N}$ and valley force of $3000 \mathrm{~N}$ are shown in Figure 11 (specimen \#2, current $I=4.0$ A, sampling frequency $f_{s}=0.385 \mathrm{~Hz}$ ). The relative change in signal $V_{I}$ was compared with the dimensionless parameter $V_{\text {norm }}$ obtained according to equation (16). It can be observed from Figure 11 that a noise signal is superimposed on the signal $V_{I 1} / V_{I 1 \_}$, and there is also a slow signal drift, which is the aforementioned multiplicative interference signal. The noise on the signal $V_{\text {norm }}$ is significantly reduced, and the multiplicative interference signal is eliminated. The final processing effect also indirectly proves that the DCPD signal model proposed in this study is reasonable. 


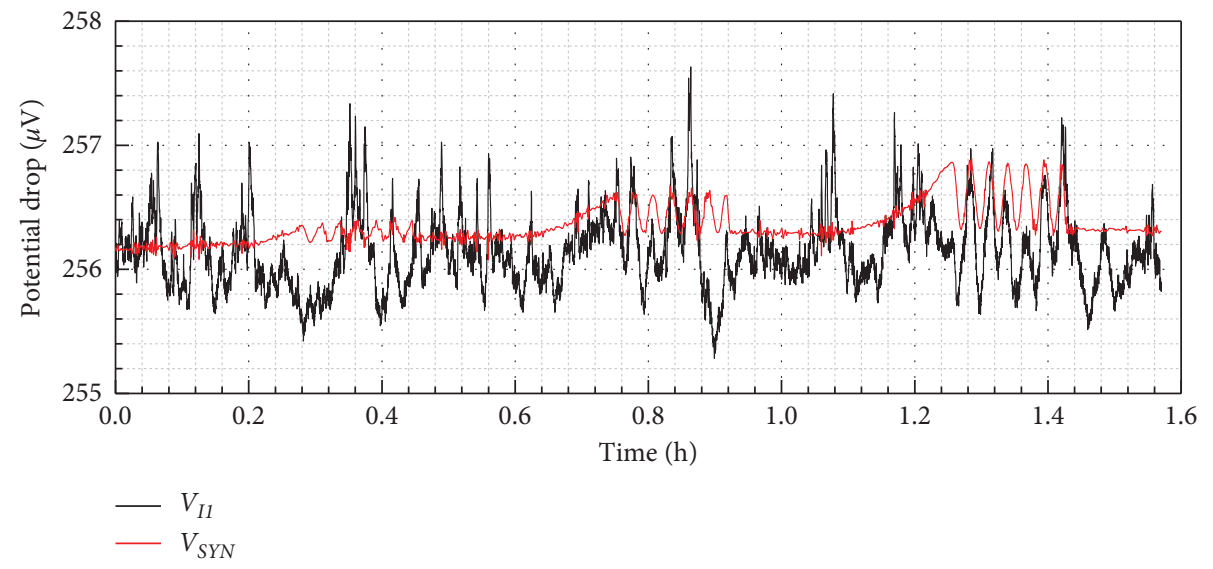

FIGURE 9: Signal processing result of DCPD signal under sinusoidal load (specimen \#2, current $I=4.0 \mathrm{~A}$ sampling frequency $f_{s}=1.295 \mathrm{~Hz}$ ).

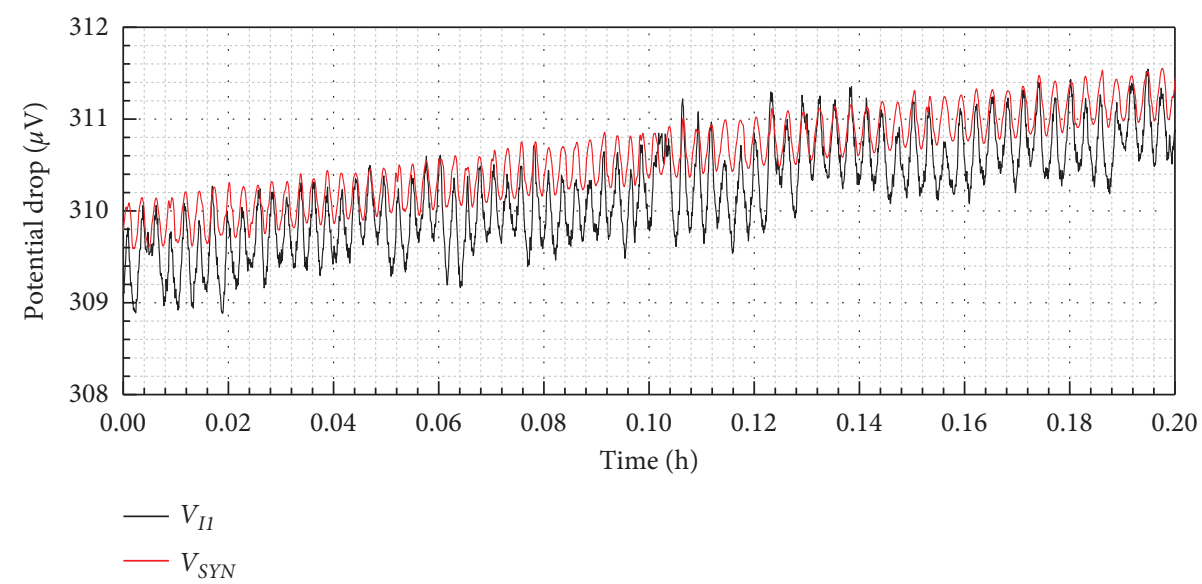

FIGURE 10: Signal processing result of DCPD signal during crack growth (specimen \#2, current $I=4.0 \mathrm{~A}$ sampling frequency $f_{s}=3.547 \mathrm{~Hz}$ ).

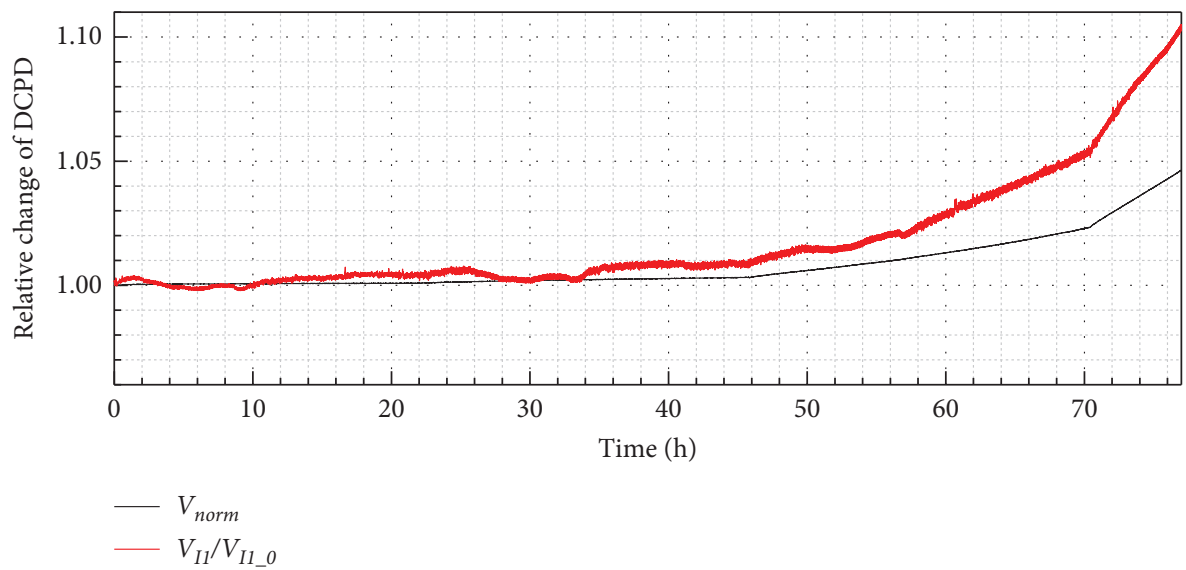

Figure 11: Comparison of $V_{I I} / V_{I O}$ and $V_{\text {norm }}$ during crack growth (specimen \#2, current $I=4.0 \mathrm{~A}$ sampling frequency $f_{s}=0.385 \mathrm{~Hz}$ ).

\section{Conclusions}

In this study, a signal processing approach for weak DCPD signals that combines wavelet threshold denoising and a variable current signal synthesis technique was proposed. According to the proposed DCPD signal composition model, the principle and feasibility of the proposed method were discussed theoretically. Using the static crack test and crack growth test data of $316 \mathrm{~L}$ stainless steel $\mathrm{C}(\mathrm{T})$ specimens, the anti-interference effect of the signal processing approach was analyzed. The following conclusions can be drawn: 
(1) The interference signal superimposed on the weak DCPD signal is composed of an additive TEMF, multiplicative interference signal, and random noise. The signal processing approach designed according to the three interference signals in this model can significantly reduce the amplitude of the interference signal, which shows that the signal composition model is reasonable.

(2) The proposed wavelet threshold denoising method based on Stein's unbiased risk estimate and adopting a soft threshold strategy can significantly reduce random interference signals and improve the SNR. Combined with the proposed variable current signal synthesis technique, the time-varying TEMF interference signal superimposed on the signal is significantly reduced. The multiplicative interference signal can be effectively eliminated using the reference potential method. Therefore, the proposed signal processing approach can effectively improve the accuracy and resolution of the crack growth measurements.

(3) The crack growth monitoring experiment based on the DCPD technique has a wide range of applications (including fatigue crack growth tests, stress corrosion crack growth tests, and creep crack growth tests), and the test environment is complex. The proposed signal processing approach applies wavelet threshold denoising to weak DCPD signals and proposes new ideas for signal processing based on the DCPD crack growth monitoring technique. After adjusting the parameters of this method according to the specific experimental environment, the proposed method is expected to be applied to various crack growth monitoring tests to improve the crack growth accuracy.

\section{Data Availability}

The data used to support the findings of this study are available from the corresponding author upon request.

\section{Conflicts of Interest}

The authors declare that there are no conflicts of interest regarding the publication of this paper.

\section{Acknowledgments}

This work was supported by the National Natural Science Foundation of China (Grant nos. 52075434 and 51775427) and the Natural Science Basic Research Plan in Shaanxi Province of China (Grant no. 2020JM-523). The authors would like to thank Editage for English language editing.

\section{References}

[1] D. U. Dong-Hai, C. Kai, and Y. U. Lun, "Measurement of fatigue crack growth rate of reactor structural material in air based on DCPD method," Atomic Energy Science and Technology, vol. 48, no. 8, pp. 1386-1391, 2014.
[2] D. H. Yoon, J. H. Kim, H. K. Kim, and D. H. Kim, "Fatigue crack growth characteristics for powder nickel based metallurgical superalloys at elevated temperatures," Materialwissenschaft und Werkstofftechnik, vol. 49, no. 5, pp. 522-529, 2018.

[3] K. Chen, D. U. Donghai, L. U. Hui, and L. Zhang, "Corrosion fatigue crack growth behavior of 304L stainless steel in high temperature and high pressure water," Corrosion \& Protection, vol. 39, no. 1, pp. 17-23, 2018.

[4] A. Campagnolo, G. Meneghetti, F. Berto, and K. Tanaka, "Crack initiation life in notched steel bars under torsional fatigue: synthesis based on the averaged strain energy density approach," International Journal of Fatigue, vol. 100, pp. 563-574, 2017.

[5] R. O. Ritchie and K. J. Bathe, "On the calibration of the electrical potential technique for monitoring crack growth using finite element methods," International Journal of Fracture, vol. 15, no. 1, pp. 47-55, 1979.

[6] H. H. Johnson, "Calibrating the electric potential method for studying slow crack growth," Materials Research and Standards, vol. 5, no. 1, pp. 442-445, 1965.

[7] Y. Si, J. P. Rouse, and C. J. Hyde, "Potential difference methods for measuring crack growth: a review," International Journal of Fatigue, vol. 136, p. 105624, 2020.

[8] Z. J. Li and H. Xue, "Numerical calibration of crack monitor based on direct current potential drop," Journal of Xi'an University of Science and Technology, vol. 32, no. 1, p. 116, 2012.

[9] A. Campagnolo, G. Meneghetti, F. Berto, and K. Tanaka, "Calibration of the potential drop method by means of electric FE analyses and experimental validation for a range of crack shapes," Fatigue and Fracture of Engineering Materials and Structures, vol. 41, no. 11, pp. 2272-2287, 2018.

[10] G. Meneghetti, L. Vecchiato, A. Campagnolo, P. Rech, and M. Cova, "Numerical calibration of the direct current potential drop (DCPD) method in fracture mechanics fatigue tests," Procedia Structural Integrity, vol. 28, pp. 1536-1550, 2020.

[11] W. Cai, S. Xie, C. Jomdecha et al., "Assessment of local conductivity distribution in stress corrosion crack region using direct current potential drop method," Corrosion Science, vol. 123, pp. 197-208, 2017.

[12] M. A. Hicks and A. C. Pickard, "A comparison of theoretical and experimental methods of calibrating the electrical potential drop technique for crack length determination," International Journal of Fracture, vol. 20, no. 2, pp. 91-101, 1982.

[13] N. Ramachandran, N. Arakere, and T. Goswami, "Recording of elevated temperature fatigue crack growth data by DCPD system," High Temperature Materials and Processes, vol. 19, no. 5 , pp. $357-370,2000$.

[14] A. M. Lewis, "Electromagnetic methods for NDE of metal fatigue cracks: practical techniques and theoretical models," Nondestructive Testing and Evaluation, vol. 6, no. 6, pp. 389-409, 1992.

[15] P. Ljustell, "The effect of large scale plastic deformation on fatigue crack length measurement with the potential drop method," Journal of Testing and Evaluation, vol. 39, no. 6, pp. 985-1002, 2011.

[16] K. I. Jones and P. R. Frise, "Temperature effects on DC potential drop measurements made on a zirconium alloy plate specimen,” Insight, vol. 38, no. 5, pp. 341-346, 1996.

[17] H. Xue, S. Gou, and C. Ni, "An optimal method for the layout of potential difference wiring points of the compact tensile 
specimen," Acta Electronica Sinica, vol. 48, no. 7, pp. 13961402, 2020.

[18] A. Tzabazis, A. Eisenried, D. C. Yeomans, and M. I. Hyatt, "Wavelet analysis of heart rate variability: impact of wavelet selection," Biomedical Signal Processing and Control, vol. 40, pp. 220-225, 2018.

[19] P. Chen and Q. Zhang, "Classification of heart sounds using discrete time-frequency energy feature based on S transform and the wavelet threshold denoising," Biomedical Signal Processing and Control, vol. 57, no. 1, p. 101684, 2020.

[20] D. L. Donoho, "De-noising by soft-thresholding," IEEE Transactions on Information Theory, vol. 41, no. 3, pp. 613627, 1995.

[21] L. L. Ting, J. Y. Tey, A. C. Tan, Y. J. King, and F. Abd Rahman, "Water leak location based on improved dual-tree complex wavelet transform with soft thresholding de-noising," Applied Acoustics, vol. 174, p. 107751, 2021.

[22] Y. Li, Y. Li, X. Chen, J. Yu, H. Yang, and L. Wang, "A new underwater acoustic signal denoising technique based on CEEMDAN, mutual information, permutation entropy, and wavelet threshold denoising," Entropy, vol. 20, no. 8, p. 563, 2018.

[23] Y.-x. Li and L. Wang, "A novel noise reduction technique for underwater acoustic signals based on complete ensemble empirical mode decomposition with adaptive noise, minimum mean square variance criterion and least mean square adaptive filter," Defence Technology, vol. 16, no. 3, pp. 543-554, 2020.

[24] G. A. Hartman and D. A. Johnson, "D-c electric-potential method applied to thermal/mechanical fatigue crack growth," Experimental Mechanics, vol. 27, no. 1, pp. 106-112, 1987.

[25] W. Wang, R. Dong, and W. Zeng, "A wavelet de-noising method for power quality based on an improved threshold and threshold function," Transactions of China Electrotechnical Society, vol. 34, no. 2, pp. 409-418, 2019.

[26] X. Y. Wang, J. Y. Zhang, F. Xu, and H. Gao, "A novel SchmittPPI circuit capable of resisting Gaussian noise in power Supply," Journal of Shanghai University, vol. 11, no. 1, pp. 15-19, 2005.

[27] J. Gong, "A new wavelet denoising algorithm optimized by controllable threshold function and threshold operator," Journal of Beijing University of Civil Engineering and Architecture, vol. 36, no. 2, pp. 67-73, 2020.

[28] S. G. Mallat, "A theory for multiresolution signal decomposition: the wavelet representation," IEEE Transactions on Pattern Analysis and Machine Intelligence, vol. 11, no. 7, pp. 674-693, 1989.

[29] X. Xu, M. Luo, Z. Tan, and R. Pei, "Echo signal extraction method of laser radar based on improved singular value decomposition and wavelet threshold denoising," Infrared Physics \& Technology, vol. 92, pp. 327-335, 2018. 